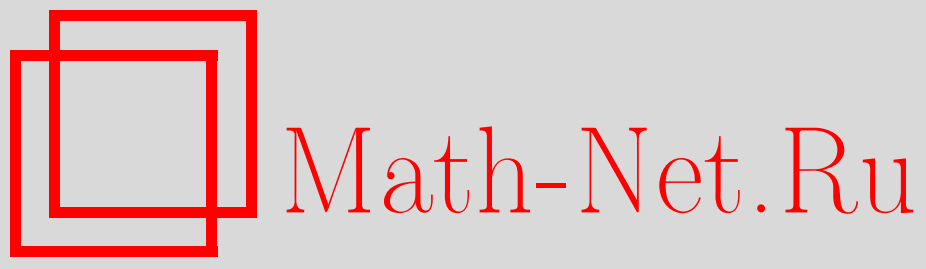

В. И. Овчинников, Интерполяционные орбиты в парах пространств Лебега, Функи. анализ и его прил., 2005, том 39, выпуск 1, 56-68

DOI: https://doi.org/10.4213/faa31

Использование Общероссийского математического портала MathNet.Ru подразумевает, что вы прочитали и согласны с пользовательским соглашением

http://www.mathnet.ru/rus/agreement

Параметры загрузки:

IP : 54.162 .27 .143

26 апреля 2023 г., 13:17:27

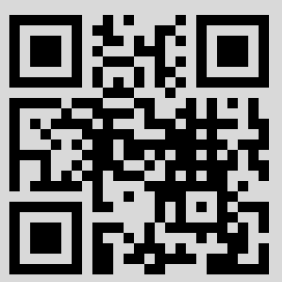


Функииональньй анализ и его приложения

2005, т. 39, вып. 1, с. 56-68

УДК 517.982

\title{
Интерполяционные орбиты в парах пространств Лебега
}

\author{
(c) 2005. В. И. Овчинников
}

Данная работа посвящена интерполяционным орбитам при действии линейных операторов из произвольной пары $\left\{L_{p_{0}}\left(U_{0}\right), L_{p_{1}}\left(U_{1}\right)\right\}$ пространств $L_{p}$ с весами в произвольную пару $\left\{L_{q_{0}}\left(V_{0}\right), L_{q_{1}}\left(V_{1}\right)\right\}$ таких же пространств, где $1 \leqslant$ $p_{0}, p_{1}, q_{0}, q_{1} \leqslant \infty$. Через $L_{p}(U)$ мы в дальнейшем обозначаем пространство измеримых функций $f$ на пространстве с мерой $\mathfrak{M}$, таких, что $f U \in L_{p}$, с нормой $\|f\|_{L_{p}(U)}=\|f U\|_{L_{p}}$. Для того чтобы описать ситуацию, введем несколько определений.

Пусть $\left\{X_{0}, X_{1}\right\}$ и $\left\{Y_{0}, Y_{1}\right\}$ - банаховы пары, $a \in X_{0}+X_{1}$. Обозначим через $\operatorname{Orb}\left(a,\left\{X_{0}, X_{1}\right\} \rightarrow\left\{Y_{0}, Y_{1}\right\}\right)$ банахово пространство таких $y \in Y_{0}+Y_{1}$, что $y=$ $T a$, где $T$ - линейный ограниченный оператор, отображающий пару $\left\{X_{0}, X_{1}\right\}$ в пару $\left\{Y_{0}, Y_{1}\right\}$. Норма определяется следующим образом:

$$
\|y\|_{\text {Orb }}=\inf _{T} \max \left(\|T\|_{X_{0} \rightarrow Y_{0}},\|T\|_{X_{1} \rightarrow Y_{1}}\right)
$$

где инфимум берется по всем представлениям $y$ в форме $y=T a$. Это пространство называется интерполяционной орбитой элемента $a$. Нашей целью будет описать пространства

$$
\operatorname{Orb}\left(a,\left\{L_{p_{0}}\left(U_{0}\right), L_{p_{1}}\left(U_{1}\right)\right\} \rightarrow\left\{L_{q_{0}}\left(V_{0}\right), L_{q_{1}}\left(V_{1}\right)\right\}\right)
$$

для всех $a$, любых $p_{0}, p_{1}, q_{0}, q_{1}, 1 \leqslant p_{0}, p_{1}, q_{0}, q_{1} \leqslant \infty$, и любых весов $U_{0}, U_{1}$, $V_{0}, V_{1}$. Данная работа содержит доказательства результатов, сформулированных в [12].

Нетривиальные результаты, касающиеся описания орбит, в отдельных случаях известны с шестидесятых годов. Ключевую роль в этих описаниях играл $K$-функционал Я. Петре. Этот функционал определяется следующим образом.

Пусть $\left\{X_{0}, X_{1}\right\}$ - банахова пара, $x \in X_{0}+X_{1}, s>0, t>0$. Положим

$$
K\left(s, t, x ;\left\{X_{0}, X_{1}\right\}\right)=\inf _{x=x_{0}+x_{1}} s\left\|x_{0}\right\|_{X_{0}}+t\left\|x_{1}\right\|_{X_{1}},
$$

где инфимум берется по всем представлениям $x$ в виде суммы $x_{0} \in X_{0}$ и $x_{1} \in X_{1}$. Функция $K(s, t)$ вогнута и однозначно определяется функцией $K(1, t, x$; $\left.\left\{X_{0}, X_{1}\right\}\right)$, которая также обозначается через $K\left(t, x ;\left\{X_{0}, X_{1}\right\}\right)$.

Если $1 \leqslant p_{0} \leqslant q_{0} \leqslant \infty, 1 \leqslant p_{1} \leqslant q_{1} \leqslant \infty$, то орбиты $\operatorname{Orb}\left(a,\left\{L_{p_{0}}\left(U_{0}\right), L_{p_{1}}\left(U_{1}\right)\right\}\right.$ $\left.\rightarrow\left\{L_{q_{0}}\left(V_{0}\right), L_{q_{1}}\left(V_{1}\right)\right\}\right)$ были описаны как обобщенные пространства Марцинкевича, т. е.

$$
\begin{aligned}
\operatorname{Orb}\left(a,\left\{L_{p_{0}}\left(U_{0}\right), L_{p_{1}}\left(U_{1}\right)\right\} \rightarrow\left\{L_{q_{0}}\left(V_{0}\right), L_{q_{1}}\left(V_{1}\right)\right\}\right) \\
\quad=M_{\varphi}\left(L_{q_{0}}\left(V_{0}\right), L_{q_{1}}\left(V_{1}\right)\right)=\left\{y: \sup _{s, t} \frac{K\left(s, t, y ;\left\{L_{q_{0}}\left(V_{0}\right), L_{q_{1}}\left(V_{1}\right)\right\}\right)}{\varphi(s, t)}<\infty\right\},
\end{aligned}
$$


где $\varphi(s, t)=K\left(s, t, a ;\left\{L_{p_{0}}\left(U_{0}\right), L_{p_{1}}\left(U_{1}\right)\right\}\right)$, для всех $a \in L_{p_{0}}\left(U_{0}\right)+L_{p_{1}}\left(U_{1}\right)$. Здесь первые результаты были получены Б. С. Митягиным и А. Кальдероном в случае $p_{0}=q_{0}=1$ и $p_{1}=q_{1}=\infty$. Решающий вклад в решение этой задачи сделали Г. Спарр в $[13,14]$ и В. И. Дмитриев в [4]. В частности, Спарр показал, что если

$$
K\left(s, t, y ;\left\{L_{p_{0}}\left(V_{0}\right), L_{p_{1}}\left(V_{1}\right)\right\}\right) \leqslant C K\left(s, t, a ;\left\{L_{p_{0}}\left(U_{0}\right), L_{p_{1}}\left(U_{1}\right)\right\}\right),
$$

для некоторой константы $C$ и всех $s, t>0$, то существует линейный оператор $T:\left\{L_{p_{0}}\left(U_{0}\right), L_{p_{1}}\left(U_{1}\right)\right\} \rightarrow\left\{L_{p_{0}}\left(V_{0}\right), L_{p_{1}}\left(V_{1}\right)\right\}$, такой, что $y=T a$.

Дмитриев [4] также нашел описание орбит в случае любых $p_{0}, p_{1}, 1 \leqslant p_{0}, p_{1}$ $\leqslant \infty$, и $q_{0}=q_{1}=1$, а также в случае любых $p_{1}, q_{0}, 1 \leqslant p_{1}, q_{0} \leqslant \infty$, и $p_{0}=$ $q_{1}=1$. Эти результаты Дмитриева были недооценены в свое время из-за того, что найденное им описание казалось неудовлетворительным.

Результаты, о которых пойдет речь в данной работе, восходят к статье [8], где были найдены оптимальные интерполяционные теоремы в классе пространств Лионса-Петре. Эти теоремы, в частности, дают описание интерполяционных орбит для элементов $a$ из специального однопараметрического семейства, но для любых $p_{0}, p_{1}, q_{0}, q_{1}, 1 \leqslant p_{0}, p_{1}, q_{0}, q_{1} \leqslant \infty$. В развитие этого подхода в [9] была сформулирована следующая гипотеза относительно орбит: пространство $\operatorname{Orb}\left(a,\left\{L_{p_{0}}\left(U_{0}\right), L_{p_{1}}\left(U_{1}\right)\right\} \rightarrow\left\{L_{q_{0}}\left(V_{0}\right), L_{q_{1}}\left(V_{1}\right)\right\}\right)$ расположено между $L_{q_{0}}\left(V_{0}\right)$ и $L_{q_{1}}\left(V_{1}\right)$ в точности в том же месте, в котором расположено пространство Кальдерона-Лозановского $\varphi\left(L_{r_{0}}\left(W_{0}\right), L_{r_{1}}\left(W_{1}\right)\right)$ между пространствами $L_{r_{0}}\left(W_{0}\right)$ и $L_{r_{1}}\left(W_{1}\right)$ для любых весов $W_{0}$ и $W_{1}$, где $\varphi(s, t)=K\left(s, t, a,\left\{L_{p_{0}}\left(U_{0}\right)\right.\right.$, $\left.\left.L_{p_{1}}\left(U_{1}\right)\right\}\right)$ и $r_{0}^{-1}=\left(q_{0}^{-1}-p_{0}^{-1}\right)_{+}, r_{1}^{-1}=\left(q_{1}^{-1}-p_{1}^{-1}\right)_{+}$. Эта гипотеза была подтверждена в [10] для элементов $a$ с квазистепенной функцией $\varphi(s, t)=K(s, t, a$, $\left.\left\{L_{p_{0}}\left(U_{0}\right), L_{p_{1}}\left(U_{1}\right)\right\}\right)$. (Заметим, что пространство $L_{p_{0}}\left(U_{0}\right)+L_{p_{1}}\left(U_{1}\right)$ может не содержать таких элементов.)

В данной работе показано, что гипотеза из [9] справедлива для всех $a \in$ $L_{p_{0}}\left(U_{0}\right)+L_{p_{1}}\left(U_{1}\right)$. Кроме этого мы получим два новых описания орбит, одно из которых близко к описанию Дмитриева в [4]. Основной результат состоит в том, что $\operatorname{Orb}\left(a,\left\{L_{p_{0}}\left(U_{0}\right), L_{p_{1}}\left(U_{1}\right)\right\} \rightarrow\left\{L_{q_{0}}\left(V_{0}\right), L_{q_{1}}\left(V_{1}\right)\right\}\right)$ совпадает с пространством $\varphi\left(L_{q_{0}}\left(V_{0}\right), L_{q_{1}}\left(V_{1}\right)\right)_{r_{0}, r_{1}}$, где $\varphi\left(X_{0}, X_{1}\right)_{r_{0}, r_{1}}$ - интерполяционный функтор, обобщающий метод средних Лионса-Петре на случай функциональных параметров $\varphi$.

\section{§1. Метод средних для произвольных квазивогнутых функциональных параметров}

Пусть $\varphi(s, t)$ - интерполяционная функция, т. е. $\varphi(s, t)$ определена и является однородной функцией первой степени при $s, t>0$, а $\rho(t)=\varphi(1, t)-$ квазивогнутая функция. Предположим, что $\varphi \in \Phi_{0}$, т. е. $\varphi(1, t) \rightarrow 0$ и $\varphi(t, 1) \rightarrow 0$ при $t \rightarrow 0$. Обозначим через $\left\{t_{n}\right\}$ балансировочную последовательность для функции $\rho(t)$ (см. [3]). В терминологии [11] балансировочная последовательность - это заполняющая и равномерно разреженная последовательность для функции $\rho(t)$. Мы не станем здесь приводить всех этих определений, поскольку суть дела не пострадает, если мы воспользуемся конкретной последовательностью такого рода. Последовательность $\left\{t_{n}\right\}$ может быть построена индуктивно с помощью конструкции Осколкова-Янсона (см. [5]):

$$
t_{0}=1, \quad \min \left(\frac{\rho\left(t_{n+1}\right)}{\rho\left(t_{n}\right)}, \frac{t_{n+1} \rho\left(t_{n}\right)}{t_{n} \rho\left(t_{n+1}\right)}\right)=q>1 .
$$


Множество индексов (область определения) этой последовательности представляет собой некоторый интервал $\mathbb{M}$ в множестве целых чисел. (Для простоты в дальнейшем будем предполагать, что последовательность $\left\{t_{n}\right\}$ двусторонняя, т. е. что $n$ пробегает множество всех целых чисел.)

Основное свойство балансировочной последовательности (см. [11]) состоит в том, что

$$
K\left(s, t,\left\{\rho\left(t_{n}\right)\right\},\left\{l_{p_{0}}, l_{p_{1}}\left(t_{n}^{-1}\right)\right\}\right) \asymp \varphi(s, t)
$$

для любых $p_{0}, p_{1}, 1 \leqslant p_{0}, p_{1} \leqslant \infty$. (Как обычно, $f \asymp g$ означает, что для некоторых положительных констант $c$ и $C$ справедлива оценка $c f \leqslant g \leqslant C f$.)

ОПредЕлЕниЕ 1 . Пусть $\left\{X_{0}, X_{1}\right\}-$ произвольная банахова пара, $\varphi \in \Phi_{0}$ и $1 \leqslant p_{0}, p_{1} \leqslant \infty$. Обозначим через $\varphi\left(X_{0}, X_{1}\right)_{p_{0}, p_{1}}$ пространство таких $x \in X_{0}+X_{1}$, что

$$
\left.x=\sum_{n \in \mathbb{Z}} \rho\left(t_{n}\right) w_{n} \quad \text { (сходимость в } X_{0}+X_{1}\right),
$$

где $w_{n} \in X_{0} \cap X_{1}$ и $\left\{\left\|w_{n}\right\|_{X_{0}}\right\} \in l_{p_{0}},\left\{t_{n}\left\|w_{n}\right\|_{X_{1}}\right\} \in l_{p_{1}}$.

Норма в $\varphi\left(X_{0}, X_{1}\right)_{p_{0}, p_{1}}$ определяется естественным образом. В случае когда $\varphi(s, t)=s^{1-\theta} t^{\theta}$, где $0<\theta<1$, такие пространства были введены Лионсом и Петре и названы пространствами средних (см. [6]). В этом случае в качестве балансировочной последовательности можно взять любую геометрическую прогрессию, скажем $t_{n}=2^{n}$. Как мы увидим позднее, наше новое обозначение имеет некоторое основание.

Заметим, что пространства $\varphi\left(X_{0}, X_{1}\right)_{\infty, \infty}$ совпадают с обобщенными пространствами Марцинкевича $M_{\varphi}\left(X_{0}, X_{1}\right)$ или с пространствами $\left(X_{0}, X_{1}\right)_{\rho, \infty}$ (см. $[5,11])$.

Нашей ближайшей целью будет дать описание пространств $\varphi\left(X_{0}, X_{1}\right)_{p_{0}, p_{1}}$ в терминах $K$-функционала.

Пусть $\left\{X_{0}, X_{1}\right\}$ - пара банаховых решеток. Напомним, что пространство $\varphi\left(X_{0}, X_{1}\right)$ - это пространство всех элементов $x$ из $X_{0}+X_{1}$, таких, что $|x|=$ $\varphi\left(\left|x_{0}\right|,\left|x_{1}\right|\right)$, где $x_{0} \in X_{0}, x_{1} \in X_{1}$, с нормой

$$
\|x\|_{\varphi\left(X_{0}, X_{1}\right)}=\inf _{x_{0}, x_{1}} \max \left(\left\|x_{0}\right\|_{X_{0}},\left\|x_{1}\right\|_{X_{1}}\right),
$$

а инфимум берется по всем $x_{0}$ и $x_{1}$, таким, что $|x|$ представляется в форме $\varphi\left(\left|x_{0}\right|,\left|x_{1}\right|\right)$. Эта конструкция пространства $\varphi\left(X_{0}, X_{1}\right)$ по паре банаховых решеток $X_{0}, X_{1}$ называется конструкцией Кальдерона-Лозановского.

Лемма 1. Пусть $1 \leqslant p_{0}, p_{1} \leqslant \infty ;$ тогда

$$
\varphi\left(L_{p_{0}}\left(U_{0}\right), L_{p_{1}}\left(U_{1}\right)\right)=\varphi\left(L_{p_{0}}\left(U_{0}\right), L_{p_{1}}\left(U_{1}\right)\right)_{p_{0}, p_{1}} .
$$

(Напомним, что если $U_{0}=1$ и $U_{1}=1$, то $\varphi\left(L_{p_{0}}, L_{p_{1}}\right)$ - это пространство Орлича; см. [7] или [11].)

ДокАзАтеЛЬСтво. Рассмотрим сначала вложение

$$
\varphi\left(L_{p_{0}}\left(U_{0}\right), L_{p_{1}}\left(U_{1}\right)\right)_{p_{0}, p_{1}} \subset \varphi\left(L_{p_{0}}\left(U_{0}\right), L_{p_{1}}\left(U_{1}\right)\right) .
$$

Пусть

$$
x=\sum_{n \in \mathbb{Z}} w_{n} \rho\left(t_{n}\right)
$$


где $\left\{\left\|w_{n}\right\|_{L_{p_{0}}}\right\} \in l_{p_{0}}$ и $\left\{t_{n}\left\|w_{n}\right\|_{L_{p_{1}}}\right\} \in l_{p_{1}}$. Построим линейный оператор

$$
T:\left\{l_{p_{0}^{\prime}}, l_{p_{1}^{\prime}}\left(t_{n}^{-1}\right)\right\} \rightarrow\left\{L_{p_{0}}\left(U_{0}\right), L_{p_{1}}\left(U_{1}\right)\right\},
$$

где через $p^{\prime}$ обозначен двойственный индекс к $p$, т. е. $p^{\prime}=(1-1 / p)^{-1}$. Положим

$$
T(\xi)=\sum_{n \in \mathbb{Z}} w_{n} \xi_{n} \quad\left(\text { сходимость в } L_{p_{0}}\left(U_{0}\right)+L_{p_{1}}\left(U_{1}\right)\right)
$$

для $\xi \in l_{p_{0}^{\prime}}+l_{p_{1}^{\prime}}\left(t_{n}^{-1}\right)$.

Если $\xi \in l_{p_{0}^{\prime}}$, то ряд $\left(w_{n} \xi_{n}\right)$ абсолютно сходится в $L_{p_{0}}\left(U_{0}\right)$. Если $\xi \in l_{p_{1}^{\prime}}\left(t_{n}^{-1}\right)$, то ряд $\left(w_{n} \xi_{n}\right)=\left(w_{n} t_{n} \xi_{n} t_{n}^{-1}\right)$ абсолютно сходится в $L_{p_{1}}\left(U_{1}\right)$. Кроме того, всюду на $\mathfrak{M}$

$$
\left|\sum_{n \in \mathbb{Z}} w_{n}(m) \xi_{n}\right| \leqslant \sum_{n \in \mathbb{Z}}\left|w_{n}(m)\right|\left|\xi_{n}\right| \leqslant\left(\sum_{n \in \mathbb{Z}}\left|w_{n}(m)\right|^{p_{0}}\right)^{1 / p_{0}}\left(\sum_{n \in \mathbb{Z}}\left|\xi_{n}\right|^{p_{0}^{\prime}}\right)^{1 / p_{0}^{\prime}} .
$$

Так как

$$
\int_{\mathfrak{M}} \sum_{n \in \mathbb{Z}}\left|U_{0}(m) w_{n}(m)\right|^{p_{0}} d m=\sum_{n \in \mathbb{Z}}\left\|w_{n}\right\|_{L_{p_{0}}\left(U_{0}\right)}^{p_{0}}<\infty
$$

TO

$$
\left(\sum_{n \in \mathbb{Z}}\left|w_{n}(m)\right|^{p_{0}}\right)^{1 / p_{0}}=W_{0}(m) \in L_{p_{0}}\left(U_{0}\right)
$$

Аналогично,

$$
\left(\sum_{n \in \mathbb{Z}}\left(t_{n}\left|w_{n}(m)\right|\right)^{p_{1}}\right)^{1 / p_{1}}=W_{1}(m) \in L_{p_{1}}\left(U_{1}\right)
$$

Следовательно,

$$
\begin{array}{ll}
|T(\xi)(m)| \leqslant W_{0}(m)\|\xi\|_{l_{p_{0}^{\prime}}} & \text { для } \xi \in l_{p_{0}^{\prime}}, \\
|T(\xi)(m)| \leqslant W_{1}(m)\|\xi\|_{l_{p_{1}^{\prime}}\left(t_{n}^{-1}\right)} & \text { для } \xi \in l_{p_{1}^{\prime}}\left(t_{n}^{-1}\right) .
\end{array}
$$

Таким образом, мы получим

$$
T: l_{p_{0}^{\prime}} \rightarrow L_{\infty}\left(W_{0}^{-1}(m)\right) \subset L_{p_{0}}\left(U_{0}\right), \quad T: l_{p_{1}^{\prime}}\left(t_{n}^{-1}\right) \rightarrow L_{\infty}\left(W_{1}^{-1}(m)\right) \subset L_{p_{1}}\left(U_{1}\right) .
$$

(Без ограничения общности мы будем считать, что почти всюду на $\mathfrak{M}$ оба веса $W_{0}$ и $W_{1}$ не обращаются в нуль.)

Легко видеть, что образ последовательности $\left\{\rho\left(t_{n}\right)\right\}$ при действии оператора $T$ совпадает с $x=\sum_{n \in \mathbb{Z}} \rho\left(t_{n}\right) w_{n}$.

Ввиду соотношения (2)

$$
K\left(s, t,\left\{\rho\left(t_{n}\right)\right\},\left\{l_{p_{0}^{\prime}}, l_{p_{1}^{\prime}}\left(t_{n}^{-1}\right)\right\}\right) \asymp \varphi(s, t) .
$$

Поэтому

$$
\begin{aligned}
K(s, t, & \left.T\left(\left\{\rho\left(t_{n}\right)\right\}\right),\left\{L_{\infty}\left(W_{0}^{-1}\right), L_{\infty}\left(W_{1}^{-1}\right)\right\}\right) \\
& =K\left(s, t, x,\left\{L_{\infty}\left(W_{0}^{-1}\right), L_{\infty}\left(W_{1}^{-1}\right)\right\}\right) \leqslant C \varphi(s, t),
\end{aligned}
$$

откуда следует, что

$$
|x(m)| \leqslant K\left(W_{0}(m), W_{1}(m), x,\left\{L_{\infty}\left(W_{0}^{-1}\right), L_{\infty}\left(W_{1}^{-1}\right)\right\}\right) \leqslant C \varphi\left(W_{0}(m), W_{1}(m)\right) .
$$


Таким образом, $x \in \varphi\left(L_{p_{0}}\left(U_{0}\right), L_{p_{1}}\left(U_{1}\right)\right)$, и вложение $(3)$ доказано.

Теперь рассмотрим вложение

$$
\varphi\left(L_{p_{0}}\left(U_{0}\right), L_{p_{1}}\left(U_{1}\right)\right) \subset \varphi\left(L_{p_{0}}\left(U_{0}\right), L_{p_{1}}\left(U_{1}\right)\right)_{p_{0}, p_{1}} .
$$

Пусть $a \in \varphi\left(L_{p_{0}}\left(U_{0}\right), L_{p_{1}}\left(U_{1}\right)\right)$. Обозначим через $\psi(t) \quad K$-функционал элемента $a$, т. е.

$$
\psi(t)=K\left(t, a,\left\{L_{p_{0}}\left(U_{0}\right), L_{p_{1}}\left(U_{1}\right)\right\}\right),
$$

а через $\left\{u_{m}\right\}$ обозначим балансировочную последовательность функции $\psi$. (Заметим, что $\psi \in \Phi_{0}$, так как $a \in \varphi\left(L_{p_{0}}\left(U_{0}\right), L_{p_{1}}\left(U_{1}\right)\right) \subset\left(L_{p_{0}}\left(U_{0}\right), L_{p_{1}}\left(U_{1}\right)\right)_{\rho, \infty}$.) Тогда

$$
\psi(t) \asymp K\left(t,\left\{\psi\left(u_{m}\right)\right\},\left\{l_{p_{0}}, l_{p_{1}}\left(u_{m}^{-1}\right)\right\}\right) \asymp K\left(t,\left\{\psi\left(u_{m}\right)\right\},\left\{l_{\infty}, l_{\infty}\left(u_{m}^{-1}\right)\right\}\right) .
$$

(Напомним, что $m$, вообще говоря, пробегает некоторый интервал $\mathbb{M}$ в $\mathbb{Z}$.) По теореме Спарра [14], примененной к парам $\left\{L_{p_{0}}\left(U_{0}\right), L_{p_{1}}\left(U_{1}\right)\right\}$ и $\left\{l_{p_{0}}, l_{p_{1}}\left(u_{m}^{-1}\right)\right\}$, найдутся линейные операторы

$$
A:\left\{L_{p_{0}}\left(U_{0}\right), L_{p_{1}}\left(U_{1}\right)\right\} \rightarrow\left\{l_{p_{0}}, l_{p_{1}}\left(u_{m}^{-1}\right)\right\}
$$

и

$$
B:\left\{l_{p_{0}}, l_{p_{1}}\left(u_{m}^{-1}\right)\right\} \rightarrow\left\{L_{p_{0}}\left(U_{0}\right), L_{p_{1}}\left(U_{1}\right)\right\}
$$

такие, что $A(a)=\left\{\psi\left(u_{m}\right)\right\}$ и $B\left(\left\{\psi\left(u_{m}\right)\right\}\right)=a$. Так как конструкция Кальдерона-Лозановского является интерполяционной для пар пространств $L_{p}$ (см., например, [11]), то

$$
\left\{\psi\left(u_{m}\right)\right\} \in \varphi\left(l_{p_{0}}, l_{p_{1}}\left(u_{m}^{-1}\right)\right) .
$$

Напомним, что любой элемент $a \in \varphi\left(L_{p_{0}}\left(U_{0}\right), L_{p_{1}}\left(U_{1}\right)\right)$ принадлежит орбите последовательности $\left\{\rho\left(t_{n}\right)\right\}$ при действии линейных операторов из пары $\left\{l_{\infty}, l_{\infty}\left(t_{n}^{-1}\right)\right\}$ в пару $\left\{L_{p_{0}}\left(U_{0}\right), L_{p_{1}}\left(U_{1}\right)\right\}$ (см., например, [11]). Это значит, что существует линейный оператор $T:\left\{l_{\infty}, l_{\infty}\left(t_{n}^{-1}\right)\right\} \rightarrow\left\{L_{p_{0}}\left(U_{0}\right), L_{p_{1}}\left(U_{1}\right)\right\}$, такой, что $T\left(\left\{\rho\left(t_{n}\right)\right\}\right)=a$.

Рассмотрим произведение $A T:\left\{l_{\infty}, l_{\infty}\left(t_{n}^{-1}\right)\right\} \rightarrow\left\{l_{p_{0}}, l_{p_{1}}\left(u_{m}^{-1}\right)\right\}$, которое отображает $\left\{\rho\left(t_{n}\right)\right\}$ в $\left\{\psi\left(u_{m}\right)\right\}$.

Так как $l_{p} \subset l_{\infty}$, мы можем рассмотреть оператор $A T$ как оператор, отображающий пару $\left\{l_{\infty}, l_{\infty}\left(t_{n}^{-1}\right)\right\}$ в пару $\left\{l_{\infty}, l_{\infty}\left(u_{m}^{-1}\right)\right\}$. Вложения $l_{p} \subset l_{\infty}$ являются $(1, p)$-суммирующими операторами по теореме Карла-Беннета [1]. Напомним, что линейный оператор $F: X \rightarrow Y$ называется $(1, p)$-суммирующим оператором из банахова пространства $X$ в банахово пространство $Y$, если для любого безусловно сходящегося ряда $\left(x_{n}\right)$ в пространстве $X$ последовательность $\left\{\left\|F\left(x_{n}\right)\right\|_{Y}\right\}$ принадлежит $l_{p}$. В частности, если $p=1$, то оператор $F$ называется абсолютно суммирующим. Если оператор $F: X \rightarrow Y(1, p)$-суммирующий, то $\left\{\left\|F\left(x_{n}\right)\right\|_{Y}\right\} \in l_{p}$ и для слабо безусловно сходящихся рядов $\left(x_{n}\right)$.

Поскольку образ стандартной базисной последовательности $e_{n}$ в пространстве $l_{\infty}$ при действии оператора $T$ всегда оказывается слабо безусловно сходящимся рядом в пространстве $l_{p_{0}}$, то $\left\{\left\|A T\left(e_{n}\right)\right\|_{l_{\infty}}\right\} \in l_{p_{0}}$. Аналогичным образом, $\left\{t_{n}\left\|A T\left(e_{n}\right)\right\|_{l_{\infty}\left(u_{m}^{-1}\right)}\right\} \in l_{p_{1}}$. Следовательно,

$$
\left\{\psi\left(u_{m}\right)\right\}=\sum_{n \in \mathbb{Z}} \rho\left(t_{n}\right) A T\left(e_{n}\right)=\sum_{n \in \mathbb{Z}} \rho\left(t_{n}\right) w_{n}
$$

поэтому

$$
\left\{\psi\left(u_{m}\right)\right\} \in \varphi\left(l_{\infty}, l_{\infty}\left(u_{m}^{-1}\right)\right)_{p_{0}, p_{1}} .
$$




\section{Ввиду (5)}

$$
K\left(t,\left\{\psi\left(u_{m}\right)\right\},\left\{l_{\infty}, l_{\infty}\left(u_{m}^{-1}\right)\right\}\right) \leqslant \sum_{n \in \mathbb{Z}} \rho\left(t_{n}\right) K\left(t, w_{n},\left\{l_{\infty}, l_{\infty}\left(u_{m}^{-1}\right)\right\}\right)
$$

поэтому в силу соотношения (2)

$$
K\left(t,\left\{\psi\left(u_{m}\right)\right\},\left\{l_{p_{0}}, l_{p_{1}}\left(u_{m}^{-1}\right)\right\}\right) \leqslant C \sum_{n \in \mathbb{Z}} \rho\left(t_{n}\right) K\left(t, w_{n},\left\{l_{\infty}, l_{\infty}\left(u_{m}^{-1}\right)\right\}\right) .
$$

Применим к неравенству (6) теорему о $K$-делимости (см. [2]). Напомним, что теорема о $K$-делимости утверждает, что для любой банаховой пары $\left\{X_{0}, X_{1}\right\}$ из неравенства

$$
K\left(t, a ;\left\{X_{0}, X_{1}\right\}\right) \leqslant \sum_{n=1}^{\infty} \psi_{n}(t)
$$

где $a \in X_{0}+X_{1}$, а $\psi_{n}(t)$ - квазивогнутые функции, вытекает существование последовательности $a_{n} \in X_{0}+X_{1}$, такой, что

$$
\left.a=\sum_{n=1}^{\infty} a_{n} \quad \text { (сходимость в } X_{0}+X_{1}\right) \quad \text { и } \quad K\left(t, a_{n} ;\left\{X_{0}, X_{1}\right\}\right) \leqslant 6 \psi_{n}(t) \text {. }
$$

Из $K$-делимости для пар $\left\{l_{p_{0}}, l_{p_{1}}\left(u_{m}^{-1}\right)\right\}$ и неравенства (6) следует, что существует последовательность $w_{n}^{\prime} \in l_{p_{0}}+l_{p_{1}}\left(u_{m}^{-1}\right)$, такая, что

$$
\left\{\psi\left(u_{m}\right)\right\}=\sum_{n \in \mathbb{Z}} \rho\left(t_{n}\right) w_{n}^{\prime},
$$

И

$$
K\left(t, w_{n}^{\prime},\left\{l_{p_{0}}, l_{p_{1}}\left(u_{m}^{-1}\right)\right\}\right) \leqslant 6 C K\left(t, w_{n},\left\{l_{\infty}, l_{\infty}\left(u_{m}^{-1}\right)\right\}\right) .
$$

Так как

$$
\lim _{t \rightarrow \infty} K\left(t, w_{n}^{\prime},\left\{l_{p_{0}}, l_{p_{1}}\left(u_{m}^{-1}\right)\right\}\right)=\left\|w_{n}^{\prime}\right\|_{l_{p_{0}}}
$$

И

$$
\lim _{t \rightarrow 0} \frac{1}{t} K\left(t, w_{n}^{\prime},\left\{l_{p_{0}}, l_{p_{1}}\left(u_{m}^{-1}\right)\right\}\right)=\left\|w_{n}^{\prime}\right\|_{l_{p_{1}}\left(u_{m}^{-1}\right)},
$$

из неравенств (7) следует, что

$$
\left\|w_{n}^{\prime}\right\|_{l_{p_{0}}} \leqslant 6 C\left\|w_{n}^{\prime}\right\|_{l_{\infty}}, \quad\left\|w_{n}^{\prime}\right\|_{l_{p_{1}}\left(u_{m}^{-1}\right)} \leqslant 6 C\left\|w_{n}^{\prime}\right\|_{l_{\infty}\left(u_{m}^{-1}\right)} .
$$

Значит,

$$
\left\{\psi\left(u_{m}\right)\right\} \in \varphi\left(l_{p_{0}}, l_{p_{1}}\left(u_{m}^{-1}\right)\right)_{p_{0}, p_{1}} .
$$

Конструкция $\varphi\left(X_{0}, X_{1}\right)_{p_{0}, p_{1}}$, очевидно, является интерполяционным функтором; поэтому образ последовательности $\left\{\psi\left(u_{m}\right)\right\}$ при действии линейного оператора $B:\left\{l_{p_{0}}, l_{p_{1}}\left(u_{m}^{-1}\right)\right\} \rightarrow\left\{L_{p_{0}}\left(U_{0}\right), L_{p_{1}}\left(U_{1}\right)\right\}$ принадлежит $\varphi\left(L_{p_{0}}\left(U_{0}\right), L_{p_{1}}\left(U_{1}\right)\right)_{p_{0}, p_{1}}$. То есть $a=B\left(\left\{\psi\left(u_{m}\right)\right\}\right) \in \varphi\left(L_{p_{0}}\left(U_{0}\right), L_{p_{1}}\left(U_{1}\right)\right)_{p_{0}, p_{1}}$. Таким образом, вложение $(4)$ и лемма 1 доказаны.

ЗАмЕчАниЕ 1 . Вложение (4) в случае $p_{0}, p_{1} \geqslant 2$ можно доказать без теоремы Карла-Беннета и $K$-делимости. Мы просто можем заметить, что в пространствах $L_{p}$ из безусловной сходимости ряда $\left(w_{n}\right)$ следует, что $\left\{\left\|w_{n}\right\|_{L_{p}}\right\} \in l_{p}$, если $p \geqslant 2$.

ЗАмЕчАНИЕ 2. Легко видеть, что $a \in \varphi\left(L_{p_{0}}\left(U_{0}\right), L_{p_{1}}\left(U_{1}\right)\right)$ в том и только в том случае, когда $\left\{\psi\left(u_{m}\right)\right\} \in \varphi\left(l_{p_{0}}, l_{p_{1}}\left(u_{m}^{-1}\right)\right)$, где $\left\{u_{m}\right\}-$ балансировочная последовательность для функции $\psi(t)=K\left(t, a,\left\{L_{p_{0}}\left(U_{0}\right), L_{p_{1}}\left(U_{1}\right)\right\}\right.$. 
Напомним, что интерполяционная функция $\varphi$ называется невырожденной, если области значений функций $\varphi(t, 1)$ и $\varphi(1, t)$ при $t>0$ совпадают с $(0, \infty)$. Именно в этом случае балансировочная последовательность функции $\rho(t)=$ $\varphi(1, t)$ оказывается двусторонней.

ЛЕмма 2. Если $\varphi$ невырожденна, то пространство $\varphi\left(X_{0}, X_{1}\right)_{p_{0}, p_{1}}$ состоит из $x \in X_{0}+X_{1}$, для которых

$$
\left\{K\left(u_{m}, x,\left\{X_{0}, X_{1}\right\}\right\} \in \varphi\left(l_{p_{0}}, l_{p_{1}}\left(u_{m}^{-1}\right)\right),\right.
$$

где $\left\{u_{m}\right\}$ - балансировочная последовательность для функиии $K\left(t, x,\left\{X_{0}, X_{1}\right\}\right)$.

ЗАмечание 3 . Лемма 2 , в частности, говорит о том, что пространства $\varphi\left(X_{0}, X_{1}\right)_{p_{0}, p_{1}}$ не зависят на самом деле от выбора балансировочной последовательности функции $\rho(t)=\varphi(1, t)$.

ЗАмечАНИЕ 4 . Заметим, что пространство $\varphi\left(X_{0}, X_{1}\right)_{p, p}$ совпадает с пространством $\left(X_{0}, X_{1}\right)_{\rho, p}$, введенным С. Янсоном. Лемма 2 дает нам новое описание этих пространств.

ДокАЗАТЕЛЬСтво ЛЕммы 2. Покажем сначала, что лемма 2 справедлива для любой интерполяционной функции $\varphi \in \Phi_{0}$ и любой относительно полной банаховой пары. Напомним, что банахова пара $\left\{X_{0}, X_{1}\right\}$ называется относительно полной, если $X_{0}=\widetilde{X}_{0}$ и $X_{1}=\widetilde{X}_{1}$, где $\widetilde{X}$ обозначает пополнение пространства $X$ относительно $X_{0}+X_{1}$.

Если $x \in \varphi\left(X_{0}, X_{1}\right)_{p_{0}, p_{1}}$, то

где

$$
x=\sum_{n} \rho\left(t_{n}\right) w_{n}
$$

$$
\sum_{n}\left\|w_{n}\right\|_{X_{0}}^{p_{0}}<\infty, \quad \sum_{n}\left(t_{n}\left\|w_{n}\right\|_{X_{1}}\right)^{p_{1}}<\infty .
$$

Из разложения (8) следует неравенство

$$
K\left(t, x,\left\{X_{0}, X_{1}\right\}\right) \leqslant \sum_{n} \rho\left(t_{n}\right) K\left(t, w_{n},\left\{X_{0}, X_{1}\right\}\right) .
$$

Вновь положим $\psi(t)=K\left(t, x,\left\{X_{0}, X_{1}\right\}\right)$. Тогда

$$
K\left(t,\left\{\psi\left(u_{m}\right)\right\},\left\{l_{p_{0}}, l_{p_{1}}\left(u_{m}^{-1}\right)\right\}\right) \leqslant \sum_{n} \rho\left(t_{n}\right) K\left(t, w_{n},\left\{X_{0}, X_{1}\right\}\right) .
$$

Ввиду $K$-делимости пары $\left\{l_{p_{0}}, l_{p_{1}}\left(u_{m}^{-1}\right)\right\}$ существует последовательность $w_{n}^{\prime} \in$ $l_{p_{0}}+l_{p_{1}}\left(u_{m}^{-1}\right)$, такая, что

и

$$
\left\{\psi\left(u_{m}\right)\right\}=\sum_{n} \rho\left(t_{n}\right) w_{n}^{\prime}
$$

$$
K\left(t, w_{n}^{\prime},\left\{l_{p_{0}}, l_{p_{1}}\left(u_{m}^{-1}\right)\right\}\right) \leqslant 6 C K\left(t, w_{n},\left\{X_{0}, X_{1}\right\}\right) \text { для всех } n .
$$

В силу (9) получим

$$
\left\|w_{n}^{\prime}\right\|_{l_{p_{0}}} \leqslant 6 C\left\|w_{n}\right\|_{X_{0}}, \quad\left\|w_{n}^{\prime}\right\|_{l_{p_{1}}\left(u_{m}^{-1}\right)} \leqslant 6 C\left\|w_{n}\right\|_{X_{0}}
$$

так же, как это было в лемме 1 . Поэтому $\left\{\psi\left(u_{m}\right)\right\} \in \varphi\left(l_{p_{0}}, l_{p_{1}}\left(u_{m}^{-1}\right)\right)_{p_{0}, p_{1}}$ и $\left\{\psi\left(u_{m}\right)\right\} \in \varphi\left(l_{p_{0}}, l_{p_{1}}\left(u_{m}^{-1}\right)\right)$ по лемме 1 . 
Теперь предположим, что $\left\{\psi\left(u_{m}\right)\right\} \in \varphi\left(l_{p_{0}}, l_{p_{1}}\left(u_{m}^{-1}\right)\right)=\varphi\left(l_{p_{0}}, l_{p_{1}}\left(u_{m}^{-1}\right)\right)_{p_{0}, p_{1}}$. Тогда

$$
\left\{\psi\left(u_{m}\right)\right\}=\sum_{n} \rho\left(t_{n}\right) w_{n}^{\prime}
$$

где

$$
\left\{\left\|w_{n}^{\prime}\right\|_{l_{p_{0}}}\right\} \in l_{p_{0}}, \quad\left\{\left\|w_{n}^{\prime}\right\|_{l_{p_{1}}\left(u_{m}^{-1}\right)}\right\} \in l_{p_{1}} .
$$

Следовательно,

$$
\begin{aligned}
K\left(t, a,\left\{X_{0}, X_{1}\right\}\right) & =\psi(t) \leqslant K\left(t,\left\{\psi\left(u_{m}\right)\right\},\left\{l_{p_{0}}, l_{p_{1}}\left(u_{m}^{-1}\right)\right\}\right) \\
& \leqslant C \sum_{n} \rho\left(t_{n}\right) K\left(t, w_{n},\left\{l_{p_{0}}, l_{p_{1}}\left(u_{m}^{-1}\right)\right\}\right) .
\end{aligned}
$$

В силу $K$-делимости пары $\left\{X_{0}, X_{1}\right\}$ существует последовательность $w_{n} \in$ $X_{0}+X_{1}$, такая, что

$$
a=\sum_{n} \rho\left(t_{n}\right) w_{n} \quad \text { и } \quad K\left(t, w_{n},\left\{X_{0}, X_{1}\right\}\right) \leqslant 6 C K\left(t, w_{n}^{\prime},\left\{l_{p_{0}}, l_{p_{1}}\left(u_{m}^{-1}\right)\right\}\right) .
$$

Поэтому

$$
\begin{aligned}
& \lim _{t \rightarrow \infty} K\left(t, w_{n},\left\{X_{0}, X_{1}\right\}\right) \leqslant 6 C\left\|w_{n}^{\prime}\right\|_{l_{p_{0}}}, \\
& \lim _{t \rightarrow 0} \frac{1}{t} K\left(t, w_{n},\left\{X_{0}, X_{1}\right\}\right) \leqslant 6 C\left\|w_{n}^{\prime}\right\|_{l_{p_{1}}\left(u_{m}^{-1}\right)} .
\end{aligned}
$$

Это значит, что

$$
\left\|w_{n}\right\|_{\widetilde{X}_{0}} \leqslant 6 C\left\|w_{n}^{\prime}\right\|_{l_{p_{0}}}, \quad\left\|w_{n}\right\|_{\widetilde{X}_{1}} \leqslant 6 C\left\|w_{n}^{\prime}\right\|_{l_{p_{1}}\left(u_{m}^{-1}\right)} .
$$

Таким образом, $a \in \varphi\left(\widetilde{X}_{0}, \widetilde{X}_{1}\right)_{p_{0}, p_{1}}$. Поэтому, если $X_{0}=\widetilde{X}_{0}$ и $X_{1}=\widetilde{X}_{1}$, лемма 2 доказана без каких-либо ограничений на функцию $\varphi \in \Phi_{0}$. Можно проверить, что

$$
\varphi\left(X_{0}, X_{1}\right)_{p_{0}, p_{1}}=\varphi\left(\widetilde{X}_{0}, \widetilde{X}_{1}\right)_{p_{0}, p_{1}}
$$

если $\varphi$ невырожденна. Лемма 2 доказана.

Пусть теперь $\varphi \in \Phi_{0}$ и $\varphi$ вырожденна. Если обе функции $\varphi(1, t)$ и $\varphi(t, 1)$ ограничены, то $\varphi(t, s) \asymp \min (s, t)$ и очевидно, что

$$
\varphi\left(X_{0}, X_{1}\right)_{p_{0}, p_{1}}=X_{0} \cap X_{1}
$$

Пусть теперь $\varphi(1, t)$ ограничена, а $\varphi(t, 1)$ - нет. В этом случае балансировочная последовательность для $\rho(t)=\varphi(1, t)$ будет односторонней. Пусть интервал $\mathbb{M}$, где она задана, совпадает с множеством неположительных целых чисел, т. е. $\left\{t_{n}\right\}_{-\infty}^{n=0}$.

Покажем, что в этом случае

$$
\varphi\left(X_{0}, X_{1}\right)_{p_{0}, p_{1}}=\left(X_{0}, X_{1}\right)_{\varphi, p_{0}, p_{1}}^{K} \cap X_{0},
$$

где через $\left(X_{0}, X_{1}\right)_{\varphi, p_{0}, p_{1}}^{K}$ обозначено пространство тех $x \in X_{0}+X_{1}$, для которых

$$
\left\{K\left(u_{m}, x,\left\{X_{0}, X_{1}\right\}\right)\right\} \in \varphi\left(l_{p_{0}}, l_{p_{1}}\left(u_{m}^{-1}\right)\right) .
$$

Мы уже показали, что

$$
\left(X_{0}, X_{1}\right)_{\varphi, p_{0}, p_{1}}^{K}=\varphi\left(\widetilde{X}_{0}, \widetilde{X}_{1}\right)_{p_{0}, p_{1}} .
$$


Из того, что последовательность $\left\{t_{n}\right\}$ односторонняя, получим

$$
\varphi\left(X_{0}, X_{1}\right)_{p_{0}, p_{1}} \subset \varphi\left(X_{0}, X_{1}\right)_{\infty, \infty} \subset X_{0} .
$$

Следовательно,

$$
\varphi\left(X_{0}, X_{1}\right)_{p_{0}, p_{1}} \subset\left(X_{0}, X_{1}\right)_{\varphi, p_{0}, p_{1}}^{K} \cap X_{0} .
$$

Если теперь $x \in\left(X_{0}, X_{1}\right)_{\varphi, p_{0}, p_{1}}^{K} \cap X_{0}$, то найдется элемент $a \in\left(l_{1}, l_{1}\left(2^{-k}\right)\right)_{\varphi, p_{0}, p_{1}}$, такой, что $x=U a$, где $U:\left\{l_{1}, l_{1}\left(2^{-k}\right)\right\} \rightarrow\left\{X_{0}, X_{1}\right\}$ (см. [2], где показано, что интерполяция из пары $\left\{l_{1}, l_{1}\left(2^{-k}\right)\right\}$ в любую банахову пару в существенном описывается $K$-методом.) Пара $\left\{l_{1}, l_{1}\left(2^{-k}\right)\right\}$ относительно полна. Поэтому в силу леммы $2 a \in \varphi\left(l_{1}, l_{1}\left(2^{-k}\right)\right)_{p_{0}, p_{1}} ;$ следовательно,

$$
a=\sum_{n=-\infty}^{0} \rho\left(t_{n}\right) a_{n},
$$

где $\left\{\left\|a_{n}\right\|_{l_{1}}\right\} \in l_{p_{0}},\left\{t_{n}\left\|a_{n}\right\|_{l_{1}\left(2^{-k}\right)}\right\} \in l_{p_{1}}$. Таким образом,

$$
x=U a=\sum_{n=-\infty}^{0} \rho\left(t_{n}\right) U\left(a_{n}\right),
$$

где $\left\{\left\|U\left(a_{n}\right)\right\|_{X_{0}}\right\} \in l_{p_{0}},\left\{t_{n}\left\|U\left(a_{n}\right)\right\|_{X_{1}}\right\} \in l_{p_{1}}$. Следовательно, $x \in \varphi\left(X_{0}, X_{1}\right)_{p_{0}, p_{1}}$.

Таким образом, мы получили описание функтора $\varphi\left(X_{0}, X_{1}\right)_{p_{0}, p_{1}}$ для любых функций $\varphi \in \Phi_{0}$. Нам в дальнейшем понадобится этот функтор для всех $\varphi \in \Phi$, потому что, если по крайней мере один из индексов $p_{0}, p_{1}$ равен бесконечности, функции $K\left(s, t, a,\left\{L_{p_{0}}\left(U_{0}\right), L_{p_{1}}\left(U_{1}\right)\right\}\right)$ не обязательно принадлежат $\Phi_{0}$.

Напомним, что любая интерполяционная функция $\varphi(s, t)$ может быть представлена в виде

$$
\varphi(s, t)=\alpha s+\beta t+\varphi_{0}(s, t),
$$

где $\varphi_{0} \in \Phi_{0}$. Если $\alpha$ или $\beta$ больше 0 , то $\varphi \notin \Phi_{0}$.

ОПРЕДЕЛЕНИЕ 2. Если $\varphi$ - произвольная интерполяционная функция, то обозначим через $\varphi\left(X_{0}, X_{1}\right)_{p_{0}, p_{1}}$ пространство

$$
\alpha X_{0}+\beta X_{1}+\varphi_{0}\left(X_{0}, X_{1}\right)_{p_{0}, p_{1}},
$$

где $\alpha X$ - пространство $X$ с нормой $\|x\|_{\alpha X}=\alpha\|x\|_{X}$, если $\alpha>0$, и $\alpha X=0$, если $\alpha=0$.

Заметим, что если и $\alpha$, и $\beta$ больше нуля, то

$$
\varphi\left(X_{0}, X_{1}\right)_{p_{0}, p_{1}}=X_{0}+X_{1}
$$

для любых $p_{0}$ и $p_{1}$.

\section{§2. Основная теорема}

TEOPEMA. Пусть $\left\{L_{p_{0}}\left(U_{0}\right), L_{p_{1}}\left(U_{1}\right)\right\} u\left\{L_{q_{0}}\left(V_{0}\right), L_{q_{1}}\left(V_{1}\right)\right\}$ - банаховъь nаpъь, где $1 \leqslant p_{0}, p_{1}, q_{0}, q_{1} \leqslant \infty, u a \in L_{p_{0}}\left(U_{0}\right)+L_{p_{1}}\left(U_{1}\right)$. Положим $\varphi(s, t)=$ $K\left(s, t, a,\left\{L_{p_{0}}\left(U_{0}\right), L_{p_{1}}\left(U_{1}\right)\right\}\right)$. Тогда

$$
\operatorname{Orb}\left(a,\left\{L_{p_{0}}\left(U_{0}\right), L_{p_{1}}\left(U_{1}\right)\right\} \rightarrow\left\{L_{q_{0}}\left(V_{0}\right), L_{q_{1}}\left(V_{1}\right)\right\}\right)=\varphi\left(L_{q_{0}}\left(V_{0}\right), L_{q_{1}}\left(V_{1}\right)\right)_{r_{0}, r_{1}},
$$
где $r_{0}^{-1}=\left(q_{0}^{-1}-p_{0}^{-1}\right)_{+} u r_{1}^{-1}=\left(q_{1}^{-1}-p_{1}^{-1}\right)_{+} \cdot\left(\right.$ Как объчно $x_{+}$означает положительную часть $x$.) 
Доказательство является комбинацией следующих ниже предложений. Мы подробно рассмотрим лишь случай $\varphi \in \Phi_{0}$. Оставшийся случай $\varphi \notin \Phi_{0}$ может быть легко сведен к случаю $\varphi \in \Phi_{0}$, например, как это сделано в [11], где аналогичная ситуация возникает при $p_{0} \leqslant q_{0}$ и $p_{1} \leqslant q_{1}$.

ПрЕДЛОЖЕНИЕ 1. Для любых $p_{0}, p_{1}, q_{0}, q_{1}, 1 \leqslant p_{0}, p_{1}, q_{0}, q_{1} \leqslant \infty$, любы весов $U_{0}, U_{1}, V_{0}, V_{1}$ и для всех $a \in L_{p_{0}}\left(U_{0}\right)+L_{p_{1}}\left(U_{1}\right)$

$$
\operatorname{Orb}\left(a,\left\{L_{p_{0}}\left(U_{0}\right), L_{p_{1}}\left(U_{1}\right)\right\} \rightarrow\left\{L_{q_{0}}\left(V_{0}\right), L_{q_{1}}\left(V_{1}\right)\right\}\right) \subset \varphi\left(L_{q_{0}}\left(V_{0}\right), L_{q_{1}}\left(V_{1}\right)\right)_{r_{0}, r_{1}} .
$$

ДокаЗАТЕЛьСтво. Пусть $b=T a$, где

$$
T:\left\{L_{p_{0}}\left(U_{0}\right), L_{p_{1}}\left(U_{1}\right)\right\} \rightarrow\left\{L_{q_{0}}\left(V_{0}\right), L_{q_{1}}\left(V_{1}\right)\right\} .
$$

Напомним, что $\rho(t)=\varphi(1, t)$. Положим $a_{\rho}=\left\{\rho\left(t_{n}\right)\right\}, \psi(u)=K\left(u, b,\left\{L_{q_{0}}\left(V_{0}\right)\right.\right.$, $\left.\left.L_{q_{1}}\left(V_{1}\right)\right\}\right)$ и $b_{\psi}=\left\{\psi\left(u_{m}\right)\right\}$, где $t_{n}$ - балансировочная последовательность для $\rho(t)$, а $u_{m}$ - балансировочная последовательность для $\psi(u)$. Так как

$$
K\left(1, t, a,\left\{L_{p_{0}}\left(U_{0}\right), L_{p_{1}}\left(U_{1}\right)\right\}\right)=\varphi(1, t)=\rho(t) \asymp K\left(1, t, a_{\rho},\left\{l_{p_{0}}, l_{p_{1}}\left(t_{n}^{-1}\right)\right\}\right),
$$

И

$$
K\left(u, b,\left\{L_{q_{0}}\left(V_{0}\right), L_{q_{1}}\left(V_{1}\right)\right\}\right)=\psi(u) \asymp K\left(u, b_{\psi},\left\{l_{q_{0}}, l_{q_{1}}\left(u_{m}^{-1}\right)\right\}\right),
$$

применив теорему Спарра к $\left\{L_{p_{0}}\left(U_{0}\right), L_{p_{1}}\left(U_{1}\right)\right\}$ и $\left\{l_{p_{0}}, l_{p_{1}}\left(t_{n}^{-1}\right)\right\}$, а также к парам $\left\{L_{q_{0}}\left(V_{0}\right), L_{q_{1}}\left(V_{1}\right)\right\}$ и $\left\{l_{q_{0}}, l_{q_{1}}\left(u_{m}^{-1}\right)\right\}$, с помощью оператора $T$ можно построить линейный оператор $S:\left\{l_{p_{0}}, l_{p_{1}}\left(t_{n}^{-1}\right)\right\} \rightarrow\left\{l_{q_{0}}, l_{q_{1}}\left(u_{m}^{-1}\right)\right\}$, такой, что $S a_{\rho}=b_{\psi}$. Отсюда следует, что

$$
b_{\psi}=\sum_{n \in \mathbb{Z}} \rho\left(t_{n}\right) S\left(e_{n}\right) \quad\left(\text { сходимость в } l_{q_{0}}+l_{q_{1}}\left(u_{m}^{-1}\right)\right),
$$

где $\left\{e_{n}\right\}$ - стандартный базис в $l_{p}$.

Рассмотрим вложения $\left\{l_{q_{0}}, l_{q_{1}}\left(u_{m}^{-1}\right)\right\} \subset\left\{l_{\infty}, l_{\infty}\left(u_{m}^{-1}\right)\right\}$. Поскольку вложения $l_{q_{i}} \subset l_{\infty}$ являются $\left(1, q_{i}\right)$-суммирующими операторами (по теореме КарлаБеннета), то так же, как и в [8], отсюда получим, что если $q_{0}<p_{0}$, то образ стандартного базиса $\left\{e_{n}\right\}$ в $l_{p_{0}}$ при действии оператора $S: l_{p_{0}} \rightarrow l_{\infty}$ является $l_{r_{0}}$-последовательностью, т. е. $\left\{\left\|S\left(e_{n}\right)\right\|_{l_{\infty}}\right\} \in l_{r_{0}}$, где $r_{0}^{-1}=q_{0}^{-1}-p_{0}^{-1}$. Аналогично, $\left\{t_{n}\left\|S\left(e_{n}\right)\right\|_{l_{\infty}\left(u_{m}^{-1}\right)}\right\} \in l_{r_{1}}$, где $r_{1}^{-1}=q_{1}^{-1}-p_{1}^{-1}$. Следовательно, в любом случае

$$
\left\{\left\|S\left(e_{n}\right)\right\|_{l_{\infty}}\right\} \in l_{r_{0}} \quad \text { и } \quad\left\{t_{n}\left\|S\left(e_{n}\right)\right\|_{l_{\infty}\left(u_{m}^{-1}\right)}\right\} \in l_{r_{1}},
$$

где $r_{0}^{-1}=\left(q_{0}^{-1}-p_{0}^{-1}\right)_{+}$и $r_{1}^{-1}=\left(q_{1}^{-1}-p_{1}^{-1}\right)_{+}$.

Согласно (10), по определению $b_{\psi} \in \varphi\left(l_{\infty}, l_{\infty}\left(u_{m}^{-1}\right)\right)_{r_{0}, r_{1}}$. В силу леммы 2 это означает, что

$$
\left\{K\left(v_{m}, b_{\psi},\left\{l_{\infty}, l_{\infty}\left(u_{m}^{-1}\right)\right\}\right)\right\} \in \varphi\left(l_{r_{0}}, l_{r_{1}}\left(v_{m}^{-1}\right)\right),
$$

где $\left\{v_{m}\right\}$ - любая балансировочная последовательность функции

$$
K\left(v, b_{\psi},\left\{l_{\infty}, l_{\infty}\left(u_{m}^{-1}\right)\right\}\right) \asymp K\left(v, b_{\psi},\left\{l_{q_{0}}, l_{q_{1}}\left(u_{m}^{-1}\right)\right\}\right)=\psi(v) .
$$

Значит, можно считать, что $v_{m}=u_{m}$, и в силу (2)

$$
\begin{aligned}
K\left(u_{m}, b,\left\{L_{q_{0}}\left(V_{0}\right), L_{q_{1}}\left(V_{1}\right)\right\}\right) & \asymp K\left(u_{m}, b_{\psi},\left\{l_{q_{0}}, l_{q_{1}}\left(u_{m}^{-1}\right)\right\}\right) \\
& \asymp K\left(u_{m}, b_{\psi},\left\{l_{\infty}, l_{\infty}\left(u_{m}^{-1}\right)\right\}\right) .
\end{aligned}
$$


Поэтому $\left\{K\left(u_{m}, b,\left\{L_{q_{0}}\left(V_{0}\right), L_{q_{1}}\left(V_{1}\right)\right\}\right)\right\} \in \varphi\left(l_{r_{0}}, l_{r_{1}}\left(u_{m}^{-1}\right)\right)$. Ввиду леммы 2 предложение доказано.

Следующие предложения посвящены обратному вложению

$$
\varphi\left(L_{q_{0}}\left(V_{0}\right), L_{q_{1}}\left(V_{1}\right)\right)_{r_{0}, r_{1}} \subset \operatorname{Orb}\left(a,\left\{L_{p_{0}}\left(U_{0}\right), L_{p_{1}}\left(U_{1}\right)\right\} \rightarrow\left\{L_{q_{0}}\left(V_{0}\right), L_{q_{1}}\left(V_{1}\right)\right\}\right) .
$$

Для любого $b \in \psi\left(L_{q_{0}}\left(V_{0}\right), L_{q_{1}}\left(V_{1}\right)\right)_{r_{0}, r_{1}}$ мы должны найти линейный оператор $T \in\left\{L_{p_{0}}\left(U_{0}\right), L_{p_{1}}\left(U_{1}\right)\right\} \rightarrow\left\{L_{q_{0}}\left(V_{0}\right), L_{q_{1}}\left(V_{1}\right)\right\}$, такой, что $b=T a$. Вновь, опираясь на теорему Спарра, заменим элемент $a$ на $a_{\rho}$, элемент $b$ на $b_{\psi}$, а исходные пары на $\left\{l_{p_{0}}, l_{p_{1}}\left(t_{n}^{-1}\right)\right\}$ и $\left\{l_{q_{0}}, l_{q_{1}}\left(u_{m}^{-1}\right)\right\}$ соответственно.

ПРЕДЛОЖЕНИЕ 2. Пусть $\left\{\psi\left(u_{m}\right)\right\} \in \varphi\left(l_{r_{0}}, l_{r_{1}}\left(u_{m}^{-1}\right)\right)$. Тогда существуют последовательности $\left\{\beta_{m}^{0}\right\} \in l_{r_{0}} u\left\{\beta_{m}^{1}\right\} \in l_{r_{1}}$, такие, что

$$
K\left(s, t,\left\{\psi\left(u_{m}\right)\right\},\left\{l_{1}\left(1 / \beta_{m}^{0}\right), l_{1}\left(1 / \beta_{m}^{1} u_{m}\right)\right\}\right) \leqslant C \varphi(s, t) .
$$

ДокАЗАТЕЛЬСтво. Так как $\left\{\psi\left(u_{m}\right)\right\} \in \varphi\left(l_{r_{0}}, l_{r_{1}}\left(u_{m}^{-1}\right)\right)$, то по определению $\psi\left(u_{m}\right)=\varphi\left(\alpha_{m}^{0}, \alpha_{m}^{1} u_{m}\right)$, где $\left\{\alpha_{m}^{0}\right\} \in l_{r_{0}},\left\{\alpha_{m}^{1}\right\} \in l_{r_{1}}$.

Рассмотрим свертку $\alpha_{m}^{0}$ с $\varepsilon_{j}^{0}$, где $\varepsilon_{j}^{0}=(1-\varepsilon)^{-j}$ при $j \leqslant 0$ и $\varepsilon_{j}^{0}=0$ при $j>0$. Положим $\beta^{0}=\varepsilon^{0} * \alpha^{0}$. Аналогично, $\beta^{1}=\varepsilon^{1} * \alpha^{1}$, где $\varepsilon_{j}^{1}=(1-\varepsilon)^{j}$ при $0 \leqslant j$ и $\varepsilon_{j}^{0}=0$ при $j<0$. Выберем $\varepsilon>0$ достаточно малым. Тогда

$$
\beta_{m+1}^{0}(1-\varepsilon) \leqslant \beta_{m}^{0}, \quad \beta_{m}^{1}(1-\varepsilon) \leqslant \beta_{m+1}^{1},
$$

$\alpha_{m}^{0} \leqslant \beta_{m}^{0}, \alpha_{m}^{1} \leqslant \beta_{m}^{1}$ и $\beta^{0} \in l_{r_{0}}, \beta^{1} \in l_{r_{1}}$.

$\mathrm{B}$ самом деле, $\varepsilon^{0}, \varepsilon^{1} \in l_{1}$; поэтому $\beta^{0} \in l_{r_{0}}, \beta^{1} \in l_{r_{1}}$ в силу свойств свертки. Поскольку $\varepsilon_{0}^{0}=\varepsilon_{0}^{1}=1$, получим $\alpha_{m}^{0} \leqslant \beta_{m}^{0}, \alpha_{m}^{1} \leqslant \beta_{m}^{1}$.

По определению

$$
\beta_{m}^{0}=\sum_{k=-\infty}^{0}(1-\varepsilon)^{k} \alpha_{m-k}^{0} .
$$

(Доопределим $\alpha^{0}$ нулем вне интервала $\mathbb{M}$.)

Так как

$$
\beta_{m+1}^{0}=\sum_{k=-\infty}^{-1}(1-\varepsilon)^{k+1} \alpha_{m-k}^{0}=(1-\varepsilon) \sum_{k=-\infty}^{-1}(1-\varepsilon)^{k} \alpha_{m-k}^{0},
$$

то $\beta_{m+1}^{0}(1-\varepsilon) \leqslant \beta_{m}^{0}$.

Аналогично,

$\beta_{m}^{1}=\sum_{k=0}^{\infty}(1-\varepsilon)^{k} \alpha_{m-k}^{1} \quad$ и $\quad \beta_{m+1}^{1}=\sum_{k=-1}^{\infty}(1-\varepsilon)^{k+1} \alpha_{m-k}^{1}=(1-\varepsilon) \sum_{k=-1}^{\infty}(1-\varepsilon)^{k} \alpha_{m-k}^{1}$.

Следовательно, $\beta_{m}^{1}(1-\varepsilon) \leqslant \beta_{m+1}^{1}$.

Рассмотрим теперь $K$-функционал последовательности $\left\{\psi\left(u_{m}\right)\right\}$, который мы собираемся оценить. Легко видеть, что

$$
K\left(s, t,\left\{\psi\left(u_{m}\right)\right\},\left\{l_{1}\left(1 / \beta_{m}^{0}\right), l_{1}\left(1 / \beta_{m}^{1} u_{m}\right)\right\}\right)=\sum_{m \in \mathbb{M}} \psi\left(u_{m}\right) \min \left(\frac{s}{\beta_{m}^{0}}, \frac{t}{\beta_{m}^{1} u_{m}}\right) .
$$

Для $s=\beta_{k}^{0}, t=\beta_{k}^{1} u_{k}$ получается неравенство

$$
K\left(\beta_{k}^{0}, \beta_{k}^{1} u_{k}\right) \leqslant \sum_{m \leqslant k} \psi\left(u_{m}\right) \frac{\beta_{k}^{0}}{\beta_{m}^{0}}+\sum_{m \geqslant k} \psi\left(u_{m}\right) \frac{\beta_{k}^{1} u_{k}}{\beta_{m}^{1} u_{m}} .
$$


В силу (1) и (11) его правая часть оценивается последовательностью, эквивалентной $\psi\left(u_{k}\right)$.

Действительно, ввиду (1)

$$
\frac{\psi\left(u_{m}\right)}{\psi\left(u_{m+1}\right)} \leqslant \frac{1}{q}, \quad \frac{u_{m}}{\psi\left(u_{m}\right)} \frac{\psi\left(u_{m+1}\right)}{u_{m+1}} \leqslant \frac{1}{q},
$$

и, согласно (11),

$$
\frac{\beta_{m+1}^{0}}{\beta_{m}^{0}} \leqslant \frac{1}{1-\varepsilon}, \quad \frac{\beta_{m}^{1}}{\beta_{m+1}^{1}} \leqslant \frac{1}{1-\varepsilon} .
$$

Следовательно,

$$
\frac{\psi\left(u_{m}\right)}{\beta_{m}^{0}} \frac{\beta_{m+1}^{0}}{\psi\left(u_{m+1}\right)} \leqslant \frac{1}{q^{\prime}}, \quad \frac{\beta_{m}^{1} u_{m}}{\psi\left(u_{m}\right)} \frac{\psi\left(u_{m+1}\right)}{\beta_{m+1}^{1} u_{m+1}} \leqslant \frac{1}{q^{\prime}},
$$

где $q^{\prime}>1$ за счет малости $\varepsilon$.

Поэтому

$$
\frac{\psi\left(u_{m}\right)}{\beta_{m}^{0}} \frac{\beta_{k}^{0}}{\psi\left(u_{k}\right)} \leqslant\left(\frac{1}{q^{\prime}}\right)^{k-m}
$$

при $m \leqslant k$ и

$$
\frac{\beta_{m}^{1} u_{m}}{\psi\left(u_{m}\right)} \frac{\psi\left(u_{k}\right)}{\beta_{k}^{1} u_{k}} \leqslant\left(\frac{1}{q^{\prime}}\right)^{m-k}
$$

при $m \geqslant k$.

(В случае когда мы рассматриваем произвольную балансировочную последовательность $\left\{u_{m}\right\}$ функции $\psi$, а не последовательность $(1)$, мы можем сразу, исходя из определения, найти такое $q>1$, что

$$
\frac{\psi\left(u_{m}\right)}{\psi\left(u_{k}\right)} \leqslant \frac{C}{q^{k-m}}
$$

при $m \leqslant k$ и

$$
\frac{u_{m}}{\psi\left(u_{m}\right)} \frac{\psi\left(u_{k}\right)}{u_{k}} \leqslant \frac{C}{q^{m-k}}
$$

при $m \geqslant k$. Поэтому и в этом случае мы получаем неравенства (12)-(13), правда, с некоторой константой.)

Отсюда следует, что

$$
\sum_{m \leqslant k} \psi\left(u_{m}\right) \frac{\beta_{k}^{0}}{\beta_{m}^{0}} \leqslant C \frac{q^{\prime}}{q^{\prime}-1} \psi\left(u_{k}\right), \quad \sum_{m \geqslant k} \psi\left(u_{m}\right) \frac{\beta_{k}^{1} u_{k}}{\beta_{m}^{1} u_{m}} \leqslant C \frac{q^{\prime}}{q^{\prime}-1} \psi\left(u_{k}\right) .
$$

Таким образом,

$$
K\left(\beta_{k}^{0}, \beta_{k}^{1} u_{k}\right) \leqslant C \frac{q^{\prime}}{q^{\prime}-1} \psi\left(u_{k}\right)=C \frac{q^{\prime}}{q^{\prime}-1} \varphi\left(\alpha_{k}^{0}, \alpha_{k}^{1} u_{k}\right) \leqslant C \frac{q^{\prime}}{q^{\prime}-1} \varphi\left(\beta_{k}^{0}, \beta_{k}^{1} u_{k}\right)
$$

следовательно,

$$
K\left(s, t,\left\{\psi\left(u_{m}\right)\right\},\left\{l_{1}\left(1 / \beta_{m}^{0}\right), l_{1}\left(1 / \beta_{m}^{1} u_{m}\right)\right\}\right) \leqslant C^{\prime} \varphi(s, t) .
$$

Предложение доказано. 
ПРЕДЛОЖЕНИЕ 3. Пусть $b_{\psi}=\left\{\psi\left(u_{m}\right)\right\} \in \varphi\left(l_{r_{0}}, l_{r_{1}}\left(u_{m}^{-1}\right)\right)$. Тогда существует линейный оператор $S:\left\{l_{p_{0}}, l_{p_{1}}\left(t_{n}^{-1}\right)\right\} \rightarrow\left\{l_{q_{0}}, l_{q_{1}}\left(u_{m}^{-1}\right)\right\}$, такой, что $S\left(a_{\rho}\right)=b_{\psi}$.

ДоКАЗАТЕЛЬСтво. Не теряя общности, мы можем предположить, что $p_{0} \geqslant q_{0}$, $p_{1} \geqslant q_{1}$. По предложению 2 найдутся последовательности $\beta^{0} \in l_{r_{0}}$ и $\beta^{1} \in l_{r_{1}}$. Рассмотрим вложения

$$
\begin{aligned}
\left\{l_{1}\left(1 / \beta_{m}^{0}\right), l_{1}\left(1 / \beta_{m}^{1} u_{m}\right)\right\} & \subset\left\{l_{p_{0}}\left(1 / \beta_{m}^{0}\right), l_{p_{1}}\left(1 / \beta_{m}^{1} u_{m}\right)\right\}, \\
\left\{l_{p_{0}}\left(1 / \beta_{m}^{0}\right), l_{p_{1}}\left(1 / \beta_{m}^{1} u_{m}\right)\right\} & \subset\left\{l_{q_{0}}, l_{q_{1}}\left(u_{m}^{-1}\right)\right\}
\end{aligned}
$$

и последовательность $b_{\psi}$.

В силу (14) и предложения 2 получим

$$
K\left(s, t,\left\{\psi\left(u_{m}\right)\right\},\left\{l_{p_{0}}\left(1 / \beta_{m}^{0}\right), l_{p_{1}}\left(1 / \beta_{m}^{1} u_{m}\right)\right\}\right) \leqslant C \varphi(s, t) .
$$

Поскольку $\varphi(s, t) \asymp K\left(s, t, a_{\rho},\left\{l_{p_{0}}, l_{p_{1}}\left(t_{n}^{-1}\right)\right\}\right)$, по теореме Спарра существует линейный оператор $S:\left\{l_{p_{0}}, l_{p_{1}}\left(t_{n}^{-1}\right)\right\} \rightarrow\left\{l_{p_{0}}\left(1 / \beta_{m}^{0}\right), l_{p_{1}}\left(1 / \beta_{m}^{1} u_{m}\right)\right\}$, отображающий $a_{\rho}$ в $b_{\psi}$.

Суперпозиция $S$ и (15) дает нужный нам линейный оператор. Таким образом, предложение 3 и теорема доказаны.

\section{ЛитеРАТУРА}

1. Bennett G. Inclusion mappings between $l_{p}$ spaces. J. Funct. Anal., 13, 20-27 (1973).

2. Brudnyi Ju. A., Krugliak N. Ya. Interpolation Functors and Interpolation Spaces. I. North Holland, Amsterdam, 1991.

3. Brudnyi Ju. A., Shteinberg A. Calderon couples of Lipschitz spaces. J. Funct. Anal., 131, 459-498 (1995).

4. Дмитриев В. И. Об интерполяции операторов в пространствах $L_{p}$. ДАН СССР, 260, 1051-1054 (1981).

5. Janson S. Minimal and maximal methods in interpolation. J. Funct. Anal., 44, 50-73 (1981).

6. Lions J.-L., Peetre J. Sur une classe d'espaces d'interpolation. Inst. Hautes Études Sci. Publ. Math., 19, 5-68 (1964).

7. Лозановский Г. Я. О некоторых банаховых структурах, II. Сиб. матем. ж., 12, 562-567 (1971).

8. Овчинников В. И. Точная интерполяционная теорема в пространствах $L_{p}$. ДАН CCCP, 272, 300-303 (1983).

9. Ovchinnikov V. I. Description of orbits. In: Israel Math. Conf. Proc., Vol. 5, 1992, pp. 291-292.

10. Ovchinnikov V.I. On the description of interpolation orbits in couples of $L_{p}$ spaces, when they are not described by the $K$-method. In: Israel Math. Conf. Proc., Vol. 5, 1992, pp. 187-206.

11. Ovchinnikov V. I. The method of orbits in interpolation theory. Math. Rep., 1, part 2, 349-516 (1984).

12. Ovchinnikov V. I. Interpolation orbits in couples of $L_{p}$ spaces. C. R. Acad. Sci. Paris, Ser. I, 334, 881-884 (2002).

13. Sparr G. Interpolation des espaces $L_{w}^{p}$. C. R. Acad. Sci. Paris, Ser. A-B, 278, 491-492 (1974).

14. Sparr G. Interpolation of weighted $L_{p}$ spaces. Studia Math., 62, 229-271 (1978).

Воронежский государственный университет email: vio@func.vsu.ru
Поступило в редакцию 11 марта 2003 г. 